\title{
Health-related quality of life burden in scleroderma patients treated with two different intravenous iloprost regimens
}

\author{
T. Schioppo ${ }^{1,4}$, L. Scalone ${ }^{2,3}$, P. Cozzolino ${ }^{2}$, L. Mantovani ${ }^{2,3}$, G. Cesana ${ }^{2}$, \\ O. De Lucia1, A. Murgo', F. Ingegnoli, \\ ${ }^{1}$ Division of Clinical Rheumatology, ASST Pini-CTO, Milano, Italy; ${ }^{2}$ Centro di Studio e Ricerca \\ sulla Sanità Pubblica (CESP), Università degli Studi di Milano Bicocca, Monza, Italy; ${ }^{3}$ Fondazione CHARTA, \\ Milano, Italy; ${ }^{4}$ Department of Clinical Sciences \& Community Health, Università degli Studi di Milano, Italy
}

\begin{abstract}
SUMMARY
Systemic sclerosis (SSc)-related Raynaud's phenomenon (RP) and digital ulcers (DU) can impair health-related quality of life (HRQoL). The aim of our study was to estimate HRQoL in SSc patients treated with two different intravenous (IV) iloprost (ILO) regimens and in patients not treated with IV ILO.

96 consecutive SSc patients were enrolled in a pragmatic, prospective and non-randomized study, and divided into 3 groups: not requiring therapy with IV ILO (N=52), IV ILO once monthly (N=24) or IV ILO for 5 consecutive days every 3 months $(\mathrm{N}=20)$. Patients were followed up for three months. We assessed HRQoL using the generic preference-based questionnaire EQ-5D-5L. We conducted multiple regression analyses to estimate, in each treatment group, the mean general health $(\mathrm{GH})$ and the mean utility index of the EQ-5D-5L, adjusting for possible confounders.

The mean adjusted utility index and GH score, after three months' follow-up, were not different in the three groups: IV ILO was able to make patients requiring IV ILO similar to those not requiring it. Moreover, there was no difference in this model between the two ILO regimens (1 day monthly vs 5 consecutive days every 3 months).

The two different IV ILO regimens (the most appropriate regimen was decided according to patients' characteristics and needs) were able to stabilize HRQoL in RP secondary to SSc non-adequately controlled by oral therapy.
\end{abstract}

Key words: Iloprost; prostacyclin; systemic sclerosis; health related quality of life; EQ-5D-5L.

Reumatismo, 2019; 71 (2): 62-67

\section{INTRODUCTION}

ystemic sclerosis $(\mathrm{SSc})$ is a multi-organ immune-mediated disease leading to skin and internal organ fibrosis (1). In these patients, Raynaud's phenomenon (RP), digital ulcers (DU) and major internal organs involvement (e.g. lung) have an impact on function, work ability, social participation, body image dissatisfaction and the overall quality of life $(2,3)$. According to the recently revised European League Against Rheumatism (EULAR) recommendations for SSc treatment, dihydropyridine-type calcium antagonists should be used as first line therapy for RP and PDE-5 inhibitors can also be considered. Intravenous (IV) prostanoids, in particularly iloprost (ILO), should be used after oral therapy has failed to control severe RP (4). Furthermore, IV ILO is indicated as effective in healing DU and it should be considered in their treatment (4). Unfortunately, recommendations do not provide any indications about IV ILO dosage and regimen. A recent review of the literature and expert consensus provide practical suggestions about ILO usage in RP and DU in SSc patients (5). In the last decades, health-related quality of life (HRQoL) has been recognized as fundamental to evaluate disease course and therapy efficacy. The EuroQol-5 dimensions-5 levels (EQ-5D-5L) questionnaire is one of the most widely used generic preference-based instruments to assess the whole HRQoL, and its use is validated in SSc (6-8). The aim of the present study is to evaluate HRQoL among SSc patients 
treated with two different IV ILO regimens and those not treated with IV ILO.

\section{MATERIALS AND METHODS}

\section{Study design}

Briefly, between October 2016 and April 2017, all consecutive adult subjects with SSc fulfilling the 2013 EULAR classification criteria (9) were recruited at the Division of Rheumatology, ASST Pini-CTO in Milan, Italy as part of a 3-month observational, prospective, pragmatic, single centre and no-profit study that has previously been described in detail (10). The ethical committee approved this study and informed consent was obtained from all patients. This was a sub-study protocol in which HRQoL data collected during the study were examined (inclusion criteria: SSc patients aged 18 years or older referring to our rheumatology clinic between Autumn 2016 and Winter 2017; exclusion criteria: overlap syndrome, amputation of the $3^{\text {rd }}$ or $4^{\text {th }}$ finger of the dominant hand, lack of informed consent). During a comprehensive baseline evaluation and a 3 -month follow-up period, data were collected from medical history; diagnostic examinations and investigations were performed as previously described (10).

\section{Population}

Each patient was treated according to current clinical practice and stratified in three main groups: SSc patients from the outpatient clinic not requiring therapy with IV ILO (group A), SSc patients treated with IV ILO once monthly (group B), and SSc patients treated with IV ILO for 5 consecutive days every 3 months (group C). Group $\mathrm{B}$ and $\mathrm{C}$ were treated with IV ILO at the dosage of $0.5-2.0 \mathrm{ng} / \mathrm{kg} / \mathrm{min}$ for 6 consecutive hours. IV ILO therapy, according to EULAR recommendations (4) and good clinical practice, was administered only in patients with severe RP after oral therapy failure and in patients with DU. The regimen (group B vs group C) was chosen according to demographic and clinical characteristics, logistic aspects and patients' preferences.
Health related quality of life assessment The endpoint was to describe, measure and value $\mathrm{HRQoL}$ in patients treated with IV ILO administered with two different regimens (group B and C) and in patients not treated with IV ILO (group A).

HRQoL was assessed using EQ-5D-5L, telephone interview version. A telephone appointment was made with each participant some days before the interview. Group A was interviewed at baseline and after 3 months. Group B was interviewed at every monthly infusion and 15 days after that. Group $\mathrm{C}$ was interviewed before each infusion cycle, and 15 and 45 days after the first infusion. Safety was assessed with adverse events, serious adverse events, discontinuations due to adverse events, and laboratory observations.

\section{$E Q-5 D-5 L$}

EQ-5D-5L is a preference-based generic instrument for the description, measurement and valuation of health. The Italian version of the EQ-5D-5L has been used and tested for validation in some studies (11, 12). It consists of two parts. The first one is a descriptive system including five domains: mobility, self-care, usual activities, anxiety/depression and pain/discomfort. Each domain includes 5 severity levels: no problem, mild problems, moderate problems, severe problems and extreme problems. The second part of the questionnaire consists of a visual analogue scale (VAS) measuring the overall $\mathrm{GH}$, ranging from 0 (worst imaginable health state) to 100 (best imaginable health state). To calculate the utility index from the EQ-5D-5L descriptive system, the responses in the descriptive system were converted into utilities using the mapping algorithm developed by van Hout et al. and applied on the Italian social tariffs $(13,14)$.

\section{Statistical analysis}

Sociodemographic and clinical characteristics of the study sample are described with proportions for categorical data, mean as the central tendency parameter for continuous data, and minimum and maximum values as dispersion parameters. 
We conducted multiple regression analyses to compare the mean $\mathrm{GH}$ (using the linear regression analysis) and the mean utility index (using the Tobit regression analysis) between the three treatment groups, ad-

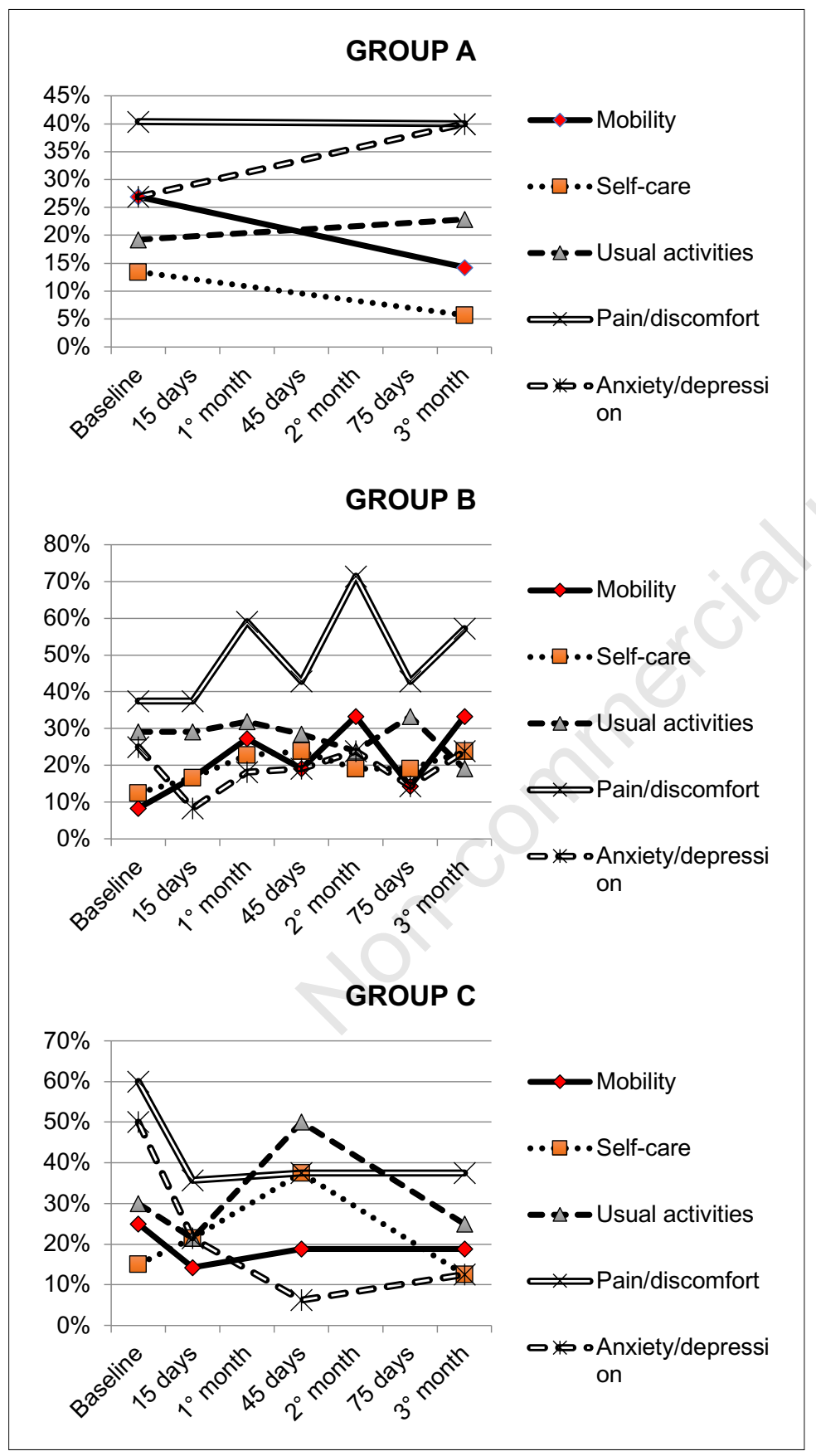

Figure 1 - Patients reporting moderate, severe or extreme problems, in every domain considered, for each treatment group (group A: no IV ILO, group B: IV ILO once monthly, group C: IV ILO for 5 consecutive days every 3 months). justing for possible confounders: age, sex, treatment group, baseline utility or $\mathrm{GH}$, average outdoor temperature during the week before the evaluation at the patient's place of residence (data provided by Meteo Operations Italia (MOPI) Srl - Centro Epson Meteo), RP VAS, disease duration and modified Rodnan skin score.

In the regression analyses, P-values $<0.05$ (two-tailed tests) were considered statistically significant and are reported with $95 \%$ confidence intervals. All analyses were conducted using Stata SE 12 (Stata Corp., College Station, TX, USA) software.

\section{RESULTS}

\section{Study population}

Between Autumn 2016 and Winter 2017, a total of $109 \mathrm{SSc}$ patients were enrolled in the study. 13 patients did not satisfy eligibility criteria and were then excluded. Among the $96 \mathrm{SSc}$ patients included, 52 were not on ILO therapy (group A), 24 were treated with IV ILO once monthly (group B), and 20 received IV ILO 5 days every 3 months (group C). Of these, for each group, respectively, 35, 21 and 16 completed the study. In group A, 17 patients were evaluated after the time limit for study follow-up; in group B there were 3 drop-outs: 1 atrial fibrillation not correlated with IV ILO, 1 pneumonia and 1 breast cancer relapse; in group $\mathrm{C}$ there were 4 drop-outs: 1 for IV ILO intolerance and 3 were evaluated after the time limit for study follow-up. Patients who did not complete the study were included in statistical analysis until their last follow-up in order to minimize distortion. The proportion of patients who discontinued treatment was similar between the two treatments. Patients had a median age of 62.2 years, $86.5 \%$ were female, $88.5 \%$ presented a cutaneous limited SSc, $97.9 \%$ were ANA positive (centromere pattern $51 \%$ ), $38.5 \%$ were anti-Scl70 positive, $16.7 \%$ were active smokers and $30.2 \%$ (group A: 23\%, B: $57 \%$, C: $44 \%$ ) had a history of one or more DU. At baseline no DU was observed and all patients had RP. Patients included in the study were treated with systemic corticosteroids (group A: 9\%, B: 38\%, C: $25 \%$ ), calcium 
Table I - EQ-5D-5L (percentages of patients reporting moderate, severe or extreme problems for each dimension considered) and general health (GH) for each treatment group (group A: no IV ILO, group B: IV ILO once monthly, group C: IV ILO for 5 consecutive days every 3 months).

\begin{tabular}{|c|c|c|c|c|c|c|c|}
\hline Group A & Baseline & 15 days & $1^{\text {st }}$ month & 45 days & $2^{\text {nd }}$ month & 75 days & $3^{\text {rd }}$ month \\
\hline Mobility & $27 \%$ & - & - & - & - & - & $14.3 \%$ \\
\hline Self-care & $13.5 \%$ & - & - & - & - & - & $5.7 \%$ \\
\hline Usual activities & $19.2 \%$ & - & - & - & - & - & $22.9 \%$ \\
\hline Pain/discomfort & $40.4 \%$ & - & - & - & - & - & $40.0 \%$ \\
\hline Anxiety/depression & $26.9 \%$ & - & - & - & - & - & $40.0 \%$ \\
\hline $\mathrm{GH}(\mathrm{VAS}) \pm \mathrm{sd}$ & $72.5 \pm 19$ & - & - & - & - & - & $78.6 \pm 16.5$ \\
\hline \multicolumn{8}{|l|}{ Group B } \\
\hline Mobility & $8 \%$ & $16.7 \%$ & $27.3 \%$ & $19.0 \%$ & $33.3 \%$ & $14.3 \%$ & $33.3 \%$ \\
\hline Self-care & $12.5 \%$ & $16.7 \%$ & $22.7 \%$ & $23.8 \%$ & $19.0 \%$ & $19.0 \%$ & $23.8 \%$ \\
\hline Usual activities & $29.2 \%$ & $29.2 \%$ & $31.8 \%$ & $28.6 \%$ & $23.8 \%$ & $33.3 \%$ & $19.0 \%$ \\
\hline Pain/discomfort & $37.5 \%$ & $37.5 \%$ & $59.1 \%$ & $42.9 \%$ & $71.4 \%$ & $42.9 \%$ & $57.1 \%$ \\
\hline Anxiety/depression & $25.0 \%$ & $8.3 \%$ & $18.2 \%$ & $19.0 \%$ & $23.8 \%$ & $14.3 \%$ & $23.8 \%$ \\
\hline $\mathrm{GH}(\mathrm{VAS}) \pm \mathrm{sd}$ & $64.8 \pm 19.2$ & $73.9 \pm 17.3$ & $67.7 \pm 18.8$ & $73.1 \pm 18.1$ & $64 \pm 18.4$ & $65 \pm 21.6$ & $66.7 \pm 19.3$ \\
\hline Group C & \multicolumn{7}{|c|}{0} \\
\hline Mobility & $25 \%$ & $14.3 \%$ & - & $18.8 \%$ & - & - & $18.8 \%$ \\
\hline Self-care & $15.0 \%$ & $21.4 \%$ & - & $37.5 \%$ & - & - & $12.5 \%$ \\
\hline Usual activities & $30.0 \%$ & $21.4 \%$ & - & $50.0 \%$ & - & - & $25.0 \%$ \\
\hline Pain/discomfort & $60.0 \%$ & $35.7 \%$ & - & $37.5 \%$ & - & - & $37.5 \%$ \\
\hline Anxiety/depression & $50.0 \%$ & $21.4 \%$ & - & $6.3 \%$ & - & - & $12.5 \%$ \\
\hline $\mathrm{GH}(\mathrm{VAS}) \pm \mathrm{sd}$ & $68 \pm 16.2$ & $65.3 \pm 14.9$ & - & $68.4 \pm 16.7$ & - & - & $75 \pm 14.6$ \\
\hline
\end{tabular}

$\mathrm{GH}$, general health; VAS, visual analogue scale; sd, standard deviation.

channel blockers (group A: 34\%, B: 57\%, C: $38 \%$ ), peripheral vasodilators (group A: $23 \%, B: 33 \%, C: 44 \%)$, endothelin receptor antagonists (group A: 9\%, B: 33\%, C: $19 \%)$ and immunosuppressive therapy (group A: 14\%, B: 24\%, C: 25\%). Detailed information on treatment and comorbidities is available in the supplementary materials of the original article (10).

\section{EQ-5D-5L and general health}

Percentages of patients who reported moderate, severe or extreme problems for every domain considered are reported in Figure 1 and Table I. In group A, the parameters considered were not different from baseline; in group B, the parameters considered (particularly pain/discomfort) were at lowest levels 15 days after IV ILO infusions; in group $\mathrm{C}$, all the parameters considered decreased 15 days after the first cycle and tended to rise till the following one.
Moreover, we described the overall trend of $\mathrm{GH}$ for each group (Figure 2 and Table I). $\mathrm{GH}$ showed an increase, even if not statistically significant, after each IV ILO infu-

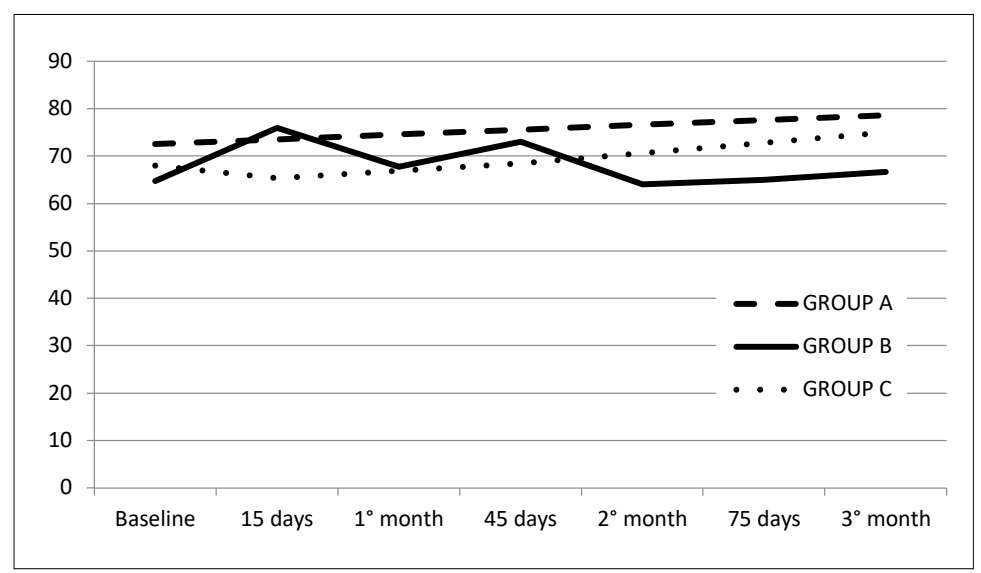

Figure 2 - General health, measured as visual analogue scale from 0 to 100, for each treatment group (group A: no IV ILO, group B: IV ILO once monthly, group C: IV ILO for 5 consecutive days every 3 months). 
Table II - Regression model for utility index and general health (GH) at the end of the three months in the three patient groups.

\begin{tabular}{|c|c|c|c|c|c|c|c|c|}
\hline \multirow{3}{*}{$\begin{array}{l}\text { Independent variable } \\
\text { description }\end{array}$} & \multicolumn{4}{|c|}{ Utility index } & \multicolumn{4}{|c|}{ GH (VAS) } \\
\hline & \multirow{2}{*}{$\begin{array}{c}\text { Coefficient } \\
-0.001\end{array}$} & \multirow{2}{*}{$\begin{array}{c}\mathbf{P} \\
0.149\end{array}$} & \multicolumn{2}{|c|}{$95 \% \mathrm{Cl}$} & \multirow{2}{*}{$\begin{array}{c}\text { Coefficient } \\
-0.332 \\
\end{array}$} & \multirow{2}{*}{$\begin{array}{c}\mathbf{P} \\
0.026\end{array}$} & \multicolumn{2}{|c|}{$95 \% \mathrm{Cl}$} \\
\hline & & & -0.002 & 0.000 & & & -0.623 & -0.041 \\
\hline \multicolumn{9}{|l|}{ Sex } \\
\hline Female (reference) & - & - & - & - & - & - & - & - \\
\hline Male & 0.028 & 0.148 & -0.010 & 0.067 & 3.637 & 0.521 & -7.618 & 14.891 \\
\hline \multicolumn{9}{|l|}{ Treatment group } \\
\hline ILO monthly (reference) & - & - & - & - & - & - & - & - \\
\hline ILO for 5 days every 3 months & 0.001 & 0.975 & -0.037 & 0.038 & 7.252 & 0.182 & -3.486 & 17.990 \\
\hline No ILO & -0.005 & 0.771 & -0.037 & 0.028 & 9.160 & 0.055 & -0.185 & 18.505 \\
\hline Utility at baseline & 0.731 & 0.000 & 0.526 & 0.936 & 0.270 & 0.012 & 0.061 & 0.478 \\
\hline Average temperature & 0.003 & 0.155 & -0.001 & 0.007 & 0.219 & 0.714 & -0.971 & 1.409 \\
\hline RP VAS & -0.005 & 0.054 & -0.010 & 0.000 & -1.114 & 0.131 & -2.569 & 0.341 \\
\hline Disease duration & 0.001 & 0.534 & -0.001 & 0.002 & 0.172 & 0.510 & -0.348 & 0.693 \\
\hline Skin score (mRSS) & -0.003 & 0.021 & -0.006 & -0.001 & -0.161 & 0.695 & -0.977 & 0.655 \\
\hline Constant & 0.241 & 0.025 & 0.032 & 0.451 & 68.969 & 0.000 & 37.504 & 100.43 \\
\hline
\end{tabular}

ILO, iloprost; VAS, visual analogue scale; CI, coefficient interval; RP, Raynaud's phenomenon; mRSS, modified Rodnan skin score.

sion in group B. GH tended to build up 15 days after IV ILO therapy for 5 consecutive days (group C).

The results of the regression models showed that $\mathrm{GH}$ and utility index were not different between the three different groups, adjusting for the possible confounders considered, as shown from the P-values $>0.5$ and 95\% confidence intervals including the 0 value (Table II).

\section{DISCUSSION}

To our knowledge, this is the first study exploring HRQoL of SSc patients managed with three different approaches according to clinical practice: those who did not receive any IV ILO treatment, and those receiving IV ILO with two different regimens, as a second line treatment to control RP or to obtain DU healing. Due to the lack of indications about IV ILO usage, the two regimens were administered considering nuanced criteria: demographic and clinical characteristics, logistic aspects and patients' preferences. For these reasons, although a direct comparison between groups was not possible since allocation was not randomly assigned, some considerations can be made. At the end of the study, both IV ILO regimens allowed to reach and to stabilize a similar HRQoL in the three groups. We reckon that the present study has some limitations such as small sample size, not fully elucidated psychometric properties of EQ-5D-5L and short follow-up.

Healthcare therapeutic interventions should include both evidence-based medicine (EBM) and shared decision-making (SDM) approaches (15). Since a clearly superior IV ILO regimen is currently not adequately supported by EBM (5), we applied predominantly the SDM approach. The best regimen was chosen by both clinicians and patients considering patients' preferences and circumstances. In this pragmatic study, patients were first stratified according to RP severity and DU presence: IV ILO therapy was proposed only to patients not adequately controlled by oral therapy. In addition, IV ILO regimen was tailored based on the abovementioned approach.

The choices we made about IV ILO regimen provide one example of how much further medicine could go in taking advantage of a more nuanced understanding of the decision-making process. Since at the 
end of the study patients showed comparable levels of HRQoL independently from the IV ILO regimen, the SDM process seems reasonable in choosing the best IV ILO therapy for each patient. Further studies are required to assess definitively the best IV ILO regimen in patients with SSc.

\section{CONCLUSIONS}

According to EULAR recommendations, prostacyclin IV therapy should be considered in patients with RP or DU secondary to SSc not adequately treated by oral therapy. In our cohort, SSc patients with DU history were mainly treated with IV ILO. Patients treated with the two different IV ILO regimens ( 1 monthly vs 5 consecutive days every 3 months) did not show any statistically significant difference with respect to HRQoL.

Acknowledgments: we thank Meteo Operations Italia (MOPI) Srl - Centro Epson Meteo for providing temperature data.

Contributions: ST, SL, FI data collecting and analyzing; ST, SL, FI manuscript writing; CP, ML, CG, DO, MA manuscript reviewing.

Conflict of interests: the authors declare no potential conflict of interest.

\section{REFERENCES}

1. Denton CP, Khanna D. Systemic sclerosis. Lancet. 2017; 390: 1685-99.

2. Pauling JD, Domsic RT, Saketkoo LA, et al. Multi-national qualitative research study exploring the patient experience of Raynaud's phenomenon in systemic sclerosis. Arthr Care Res. 2018; 70: 1373-84.

3. Pauling JD, Saketkoo LA, Matucci-Cerinic M, et al. The patient experience of Raynaud's phenomenon in systemic sclerosis. Rheumatology. 2019; 58: 18-26.

4. Kowal-Bielecka O, Fransen J, Avouac J, et al. Update of EULAR recommendations for the treatment of systemic sclerosis. Ann Rheum. Dis. 2017; 76: 1327-39.

5. Ingegnoli F, Schioppo T, Allanore Y, et al. Practical suggestions on intravenous iloprost in Raynaud's phenomenon and digital ulcer secondary to systemic sclerosis: Systematic literature review and expert consensus. Semin Arthrit Rheum. 2019; 48: 686-93.

6. Gualtierotti R, Ingegnoli F, Scalone L, et al. Feasibility, acceptability and construct validity of EQ-5D in systemic sclerosis. Swiss Med. Weekly. 2017; 146: w14394.

7. Gualtierotti R, Scalone L, Ingegnoli F, et al. [Health related quality of life assessment in patients with systemic sclerosis]. Reumatismo. 2010; 62: 210-4.

8. Kwakkenbos L, Fransen J, Vonk MC, et al. A comparison of the measurement properties and estimation of minimal important differences of the EQ-5D and SF-6D utility measures in patients with systemic sclerosis. Clin Exper Rheumatol. 2013; 31: 50-6.

9. van den Hoogen F, Khanna D, Fransen J, et al. 2013 classification criteria for systemic sclerosis: an American College of Rheumatology/ European League against Rheumatism collaborative initiative. Arthrit Rheum. 2013; 65: 2737-47.

10. Schioppo T, Orenti A, Boracchi P, et al. Acute and chronic effects of two different intravenous iloprost regimens in systemic sclerosis: a pragmatic non-randomized trial. Rheumatology. 2018; 57: 1408-16.

11. Scalone L, Ciampichini R, Fagiuoli S, et al. Comparing the performance of the standard EQ-5D 3L with the new version EQ-5D 5L in patients with chronic hepatic diseases. Qual Life Res. 2013; 22: 1707-16.

12. Scalone L, Cortesi PA, Ciampichini R, et al. Health related quality of life norm data of the general population in Italy: results using the EQ-5D-3L and EQ-5D-5L instruments. Epidemiol Biostat Public Health. 2015; 12: e11457-15.

13. Scalone L, Cortesi PA, Ciampichini R, et al. Italian population-based values of EQ-5D health states. Value Health. 2013; 16: 814-22.

14. van Hout B, Janssen MF, Feng YS, et al. Interim scoring for the EQ-5D-5L: mapping the EQ-5D-5L to EQ-5D-3L value sets. Value Health. 2012; 15: 708-15.

15. Hoffmann TC, Montori VM, Del Mar C. The connection between evidence-based medicine and shared decision making. JAMA. 2014; 312: 1295-6. 